\title{
PROFIL GANGLIONNAIRE DES MICRO CARCINOMES PAPILLAIRES DE LA THYROIDE
}

\author{
I. LAHMAR, K. MIGHRI, A. BERKAOUI, N. BEN HAMIDA, R. FDHILA, A. MOUSSA*, \\ N. DRISS. \\ SERVICE O.R.L. CHU TAHAR SFAR. MAHDIA. \\ *SERVICE D’ANATOMIE ET CYTOLOGIE PATHOLOGIQUES. CHU MONASTIR.
}

\begin{abstract}
La prise en charge des microcarcinomes papillaires de la thyroïde est controversée surtout en matière de curages ganglionnaires.

Objectif : déterminer le profil ganglionnaire des microcarcinomes papillaires de la thyroïde afin de planifier une prise en charge adéquate.

Matériel et méthodes : C'est une étude rétrospective portant sur 20 cas de microcarcinomes papillaires de la thyroïde colligés sur une période de 14 ans (1994-2007).

Résultats : il s'agissait de 18 femmes et 2 hommes d'un âge moyen de 46 ans. Les micro carcinomes étaient de découverte per-opératoire dans $50 \%$ des cas. Ils étaient unifocaux dans $80 \%$ des cas et non encapsulés dans $40 \%$ des cas. Leur taille était supérieure à $0,5 \mathrm{~cm}$ dans $35 \%$ des cas. Cinquante six pour cent des curages ganglionnaires étaient positifs. Une métastase ganglionnaire a été retrouvée dans $83 \%$ pour les tumeurs de taille supérieure à $0,5 \mathrm{~cm}$.

Conclusion : plusieurs paramètres conditionnent les métastases ganglionnaires cervicales en matière de microcarcinomes papillaires de la thyroïde. La conduite thérapeutique doit les prendre en considération.
\end{abstract}

Mots-clés : Thyroïde, Micro carcinome papillaire, curage ganglionnaire cervical.

Management of papillary microcarcinomas of thyroid gland is subject to many discussions.

Objective : Aim of this study: determinate lymph node status in these malignant tumors.

Materials and methods : this study is about 20 cases of papillary microcarcinomas of thyroid gland, operated between 1994 and 2007,

Results : Our study was about 18 women and 2 men with a middle-age of 46 years. We had found that $35 \%$ of microcarcinomas had a size higher than $0,5 \mathrm{~cm}$. Neck lymph node metastasis were present in $83 \%$ when the size of tumor was higher than $0,5 \mathrm{~cm}$.

Conclusion : Neck lymph node metastasis in micropapillary carcinomas is conditioned by many factors of which depend the therapeutic management.

Keywords : Thyroid gland, papillary microcarcinoma, neck-lymph node dissection.

\section{INTRODUCTION}

Les microcarcinomes papillaires (MCP) sont définis par l'organisation mondiale de la santé (OMS) comme étant des carcinomes papillaires de taille inférieure à 1 centimètre (1). Ils suscitent plusieurs controverses quant à la stratégie thérapeutique. Si la thyroidectomie totale constitue l'attitude de choix vis à vis de la glande thyroïde $(2,3)$, différentes attitudes sont encore adoptées vis-à-vis des chaines ganglionnaires. Certains pratiquent des curages ganglionnaires systématiques $(4,5)$ alors que d'autre sont guidés dans leurs attitudes par les caractéristiques de la tumeur comme sa taille, son caractère encapsulé et sa multifocalité $(6,7,8)$.

L'objectif de cette étude est de préciser les paramètres dont dépendent les métastases ganglionnaires des MCP en essayant de planifier une prise en charge adéquate.

MATÉRIEL ET MÉTHODES

II s'agit d'une étude rétrospective à propos de 20 cas de
MCP colligés dans le service d'ORL de l'hôpital Tahar Sfar de Mahdia Tunisie sur une période de 14 ans (19942007).

Dix neuf patients ont eu une thyroïdectomie totale et un patient a eu une lobo-isthmectomie. Cinquante pour cent des MCP ont été découverts en per opératoire ; notre attitude dans ce cas était une thyroïdectomie totale avec curage des secteurs médians (VI) avec un curage des secteurs IV des deux côtés : cette attitude a été adoptée en absence d'adénopathies palpables en pré-opératoire. Un patient a eu seulement une lobo-isthmectomie sans geste ganglionnaire.

Sept patients avaient des adénopathies cervicales palpables en préopératoire. Dans ces cas, la thyroïdectomie totale a été associée à un curage des 2 secteurs médians (VI) ainsi que des secteurs II, III, IV et V du côté de l'adénopathie et du secteur IV de l'autre côté.

Dans 50\% des cas, le MCP était découvert en postopératoire sur pièce de thyroïdectomie totale (2 cas) ou partiel- 
le (8 cas) pratiquée pour pathologie bénigne.

Dans ces cas, une totalisation de la thyroïdectomie avec curage des secteurs VI controlatéraux avec curage des secteurs IV des 2 côtés ont été réalisés dans 5 cas et un curage du secteur IV bilatéral a été réalisé dans un cas. Donc, en résumé, nous avons réalisé :

- 7 curages des secteurs II, III, IV et V d'un côté avec curage du secteur IV controlatéral et curage des secteurs VI des 2 côtés.

- 5 curages des secteurs IV des 2 côtés avec curage d'un secteur VI d'un seul côté.

- 2 curages des secteurs IV et VI des 2 côtés

- 2 curages des secteurs IV des 2 côtés.

Pour l'étude statistique, nous avons utilisé le programme SPSS avec test de X2. Ce test est statistiquement significatif pour une valeur de $p$ inférieure ou égale à 0,05 .

\section{RESULTATS}

II s'agissait de 18 femmes et 2 hommes, d'un âge moyen de 46 ans, avec des extrêmes de 24 et 75 ans.

Les tumeurs étaient de découverte postopératoire dans $50 \%$ des cas sur des pièces de thyroïdectomie pratiquées pour un nodule thyroïdien unique ou multiple. Les adénopathies cervicales palpables étaient présentes à l'examen initial dans $40 \%$ des cas. Deux cas (10\%) étaient découverts fortuitement en per-opératoire lors d'une intervention pour autre pathologie.

La taille moyenne des adénopathies révélatrices du MCP était de $4,4 \mathrm{~cm}(1-15 \mathrm{~cm})$. Ces adénopathies siégeaient dans $50 \%$ des cas (4/8) au niveau du secteur IV, dans $25 \%$ des cas (2/8) au niveau du secteur Ila, dans 12,5

$\%(1 / 8)$ au niveau du secteur III et dans $12,5 \%$ des cas au niveau du secteur $\mathrm{Vb}$. Ces adénopathies étaient uniques dans $87,5 \%$ des cas $(7 / 8)$.

Notre conduite vis-vis de la glande thyroïde était maximaliste : Une thyroïdectomie totale a été pratiquée dans 19 cas et une seule patiente a eu une lobo-isthmectomie. Les MCP étaient unifocaux dans $80 \%$ des cas (16/20), multifocaux dans les deux lobes thyroïdiens dans $15 \%$ des cas $(3 / 20)$ et multifocaux dans un lobe dans $5 \%$ des cas $(1 / 20)$.

La taille moyenne des MCP était de $0,54 \mathrm{~cm}(0,3-1 \mathrm{~cm})$. Elle était supérieure à $0,5 \mathrm{~cm}$ dans $35 \%$ des cas (7/20). Les MCP étaient non encapsulés dans $40 \%$ des cas (8/20).

Seize patients ont eu un curage ganglionnaire : 7 curages fonctionnels unilatéraux avec curages sus-claviculaires sous-omo-hyoïdiens controlatéraux et récurrentiels bilatéraux, 7 curages sus-claviculaires sous-omo-hyoïdiens et et récurrentiels bilatéraux et 2 curages récurrentiels. Les curages étaient positifs dans $56 \%$ des cas (9 patients parmi 16). Les métastases ganglionnaires étaient unilatérales dans $66 \%$ des cas et bilatérales dans $33 \%$ des cas. Le taux de métastases ganglionnaires des tumeurs de taille supérieure à $0,5 \mathrm{~cm}$ était de $83 \%$
(5 cas/6), alors que celui des tumeurs de taille inférieure à $0,5 \mathrm{~cm}$ était de $20 \%(4 \mathrm{cas} / 10)(\mathrm{p}<0,1)$

Les curages ganglionnaires étaient positifs dans $75 \%$ des cas pour les tumeurs non encapsulées, et dans $37,5 \%$ des cas pour les tumeurs encapsulées $(p=0,131)$.

Les tumeurs unifocales étaient associées à un taux de métastase ganglionnaire de $53 \%$, alors que les tumeurs multifocales étaient associées à un taux de $66 \%$ $(p=0,687)$

En présence d'adénopathies cervicales palpables à l'examen initial, les curages ganglionnaires étaient positifs dans $86 \%$ des cas (6cas/7). Par contre, seulement $33 \%$ (3 cas/9) des curages ganglionnaires étaient positifs en absence d'adénopathies $(p<0,1)$.

L'étude des différents curages a objectivé un ensemble de 191 ganglions dont 36 étaient métastatiques avec un taux de 18,8\% (36/191). Parmi ces 36 adénopathies métastatiques, 4 seulement ont été trouvées chez des patients n'ayant pas d'adénopathies cervicales palpables cliniquement sur un ensemble de 59 ganglions avec un taux de 6,7\% (4/59) ; tandis que 32 adénopathies métastatiques ont été présentées chez des patients ayant des adénopathies palpables, sur un ensemble de 132 ganglions avec un taux de 24,2\% (32/132) (Tableau I)

\begin{tabular}{|lc|}
\hline Nombre total des ganglions : & 191 \\
\hline Nombre total de ganglions métastatiques : & $36(18,8 \%)$ \\
\hline Nombre de N+ Histologiques pour les No cliniques : & $4(6,7 \%)$ \\
\hline Nombre de N+ Histologiques pour les N+ cliniques : & $32(24,2 \%)$ \\
\hline
\end{tabular}

\section{Tableau I : Résultats histologiques des curages} ganglionnaires

Les curages récurrentiels ont montré 18 ganglions métastatiques sur un ensemble de 50 ganglions avec un taux de $36 \%$. Les curages fonctionnels ont montré 15 ganglions métastatiques sur un ensemble de 69 ganglions, avec un taux de $21,7 \%$. Les curages sus claviculaires sous-omo-hyoïdiens n'ont montré que 3 ganglions métastatiques sur un ensemble de 72 ganglions avec un taux de $4,1 \%$.

\section{DISCUSSION}

Le diagnostic de MCP se fait le plus souvent en post opératoire après étude des pièces de thyroïdectomies totales ou partielles réalisées pour une lésion thyroïdienne bénigne. Dans notre série, $50 \%$ des MCP étaient découverts selon ce mode qui avait atteint $78,3 \%$ dans l'étude de Sturniolo et coll. (1).

Une fois le diagnostic de MCP posé, le chirurgien se trouve face à des réflexions thérapeutiques. Si la thyroïdectomie était partielle dans le premier temps opératoire, fautil totaliser ? Faut-il pratiquer un curage ganglionnaire sys- 
tématique?

Vu le bon pronostic des MCP avec mortalité à distance inférieure à $1 \%$ (9) et devant la lourde morbidité récurrentielle $(1-2 \%)$ et parathyroïdienne $(1,7 \%)$ certains auteurs se contentent d'une lobo-isthmectomie du coté de la tumeur $(9,10)$.

Cependant, la majorité des études $(1,2,3,6,7)$ soutiennent un geste radical vis-à-vis de la glande thyroïde. Les arguments en faveur de la thyroïdectomie totale (TT) sont (Tableau II) :

- La multifocalité des MCP est fréquente de même que la bilatéralité.

- Les métastases lymphatiques des MCP sont fréquentes.

- Les récidives locales sont moins fréquentes en cas de thyroïdectomie totale.

La TT expose aux mêmes risques opératoires que la thyroïdectomie partielle entre les mains d'un chirurgien expérimenté.

Si la TT est désormais l'attitude de choix face à un MCP, plusieurs controverses sont encore posées concernant l'attitude vis-à-vis des chaines ganglionnaires. II est admis que, comme le carcinome papillaire, le MCP est lymphophile avec des chiffres variant entre 6,1 et $65 \%$ dans la littérature $(3,11,12)$. Dans notre série, le taux de métastases ganglionnaires était de $56 \%$.

De même, l'envahissement ganglionnaire des MCP dépend de plusieurs paramètres liés aux caractéristiques de la tumeur elle-même qui sont la taille, la rupture capsulaire et la multifocalité.

En effet, le taux de métastases ganglionnaires est plus élevé pour les tumeurs de taille supérieure à $0,5 \mathrm{~cm}$ $(6,13)$. Dans notre série le taux de métastase ganglionnaire a atteint $83 \%$ pour les tumeurs de taille supérieure à $0,5 \mathrm{~cm}$, contre $20 \%$ pour les tumeurs de taille inférieure à $0,5 \mathrm{~cm}(p<0,1)$.

Le caractère non encapsulé est aussi un facteur prédictif de l'envahissement ganglionnaire. Les tumeurs non encapsulées dans notre série étaient associées à un taux plus élevé de métastases ganglionnaires. Ceci a été également retrouvé dans la littérature $(7,12)$.

Les tumeurs multifocales étaient responsables dans notre série d'un taux plus élevé de métastases ganglionnaires. Ito (14) a trouvé les mêmes constatations.

A coté des caractères histologiques de la tumeur, un deuxième élément fondamental conditionne la décision du chirurgien : c'est la présence ou non d'adénopathies cervicales palpables à l'examen clinique (6).

Les attitudes chirurgicales se distinguent ainsi entre maximalistes (partisans des curages de principe) et minimalistes (partisans des curages de nécessité) (tableau I).

\begin{tabular}{|c|c|}
\hline Minimalistes $(16,17,18)$ & Maximalistes $(4,5,15)$ \\
\hline $\begin{array}{l}\text {-Le pronostic est indépendant de la présence de } \\
\text { métastases ganglionnaires. } \\
\text {-Les métastases ganglionnaires des MCP ont } \\
\text { une incidence faible. } \\
\text { - Les micro foyers tumoraux ayant échappés à } \\
\text { la chirurgie peuvent être éliminés par l'iode } \\
\text { actif. } \\
\text {-Le curage ganglionnaire de principe ne réduit } \\
\text { pas le taux de récidives lymphatiques. }\end{array}$ & $\begin{array}{l}\text { Les métastases ganglionnaires sont corrélées à un } \\
\text { pronostic défavorable. } \\
\text {-Les métastases de la chaine récurrencille sont de } \\
\text { diagnostic préopératoire difficile. } \\
\text {-Le curage ganglionnaire systématique facilite la } \\
\text { surveillance post opératoire. } \\
\text {-Le traitement par l'Iode radio actif aura une } \\
\text { efficacité meilleure après un curage ganglionnaire } \\
\text { systématique. } \\
\text {-Une thyroïdectomie totale seule expose aux } \\
\text { mêmes risques opératoires qu'une TT avec curage } \\
\text { ganglionnaire. }\end{array}$ \\
\hline
\end{tabular}

Tableau II : différence entre attitudes maximalistes et minimalistes vis-à-vis des chaines ganglionnaires

Entre ces deux extrêmes, beaucoup d'auteurs adoptent une attitude intermédiaire qui est guidée par les données de l'examen clinique et anatomopathologique $(6,7,8,9)$. Le premier cas de figure se pose en présence d'adénopathies cervicales à l'examen initial. Ils optent, dans ces conditions pour un curage ganglionnaire systématique qui doit couvrir le territoire central ou récurrentiel et les secteurs latéraux (6).

Le deuxième cas de figure se présente en absence d'adénopathies cervicales palpables. Dans ce cas l'attitude sera raisonnée au cas par cas selon les caractéristiques histo-pathologiques de la tumeur. Le curage ganglionnaire central est systématique et le curage latéral sera pratiqué si le MCP est jugé invasif, soit par sa taille supérieure à $0,5 \mathrm{~cm}$, soit par son invasion capsulaire, soit par sa multifocalité $(7,8)$.

Notre attitude est intermédiaire vis-à-vis des secteurs ganglionnaires, justifiée par la taille de la tumeur, la capsule, la multifocalité et les adénopathies en pré-opératoire.

\section{CONCLUSION}

Le MCP est le plus souvent de découverte fortuite post opératoire imposant plusieurs réflexions thérapeutiques quant à l'association d'un geste ganglionnaire. En absence d'études randomisées, il nous emble que le curage lymphatique doit être raisonnée, adapté au patient, à la tumeur et à l'envahissement ganglionnaire diagnostiqué en préopératoire 


\section{REFERENCES}

1- Sturniolo G, Lo Shiavo MG. Le traitement chirurgical des microcarcinomes thyroïdiens. E-mémoires de L'Académie Nationale de Chirurgie,2004, 3(1) : 4752

2 -Pelizzo M.R, Boschin I.M, Toniato A, Piotto A. Papillary thyroid microcarcinoma ,Prognostic factors, management and outcome in 403 patients. Eur J Surg Oncol. 2006;32(10):1144-8.

3- Oueslati Z, Aloui M, Gritli S, Touati S, El-May A, Gamoudi A, Ben-Slimene F, Ladgham A. Microcarcinomes papillaires de la thyroïde. Expérience de l'institut Salah Azaiz. Rev Larygol Otol Rhinol. $2002 ; 123$ :39-42.

4-Noguchi S, Yamashita H, Murakami N. et al. Small carcinomas of the thyroid. A long term follow-up of 867 patients. Arch Surg. : 1996 ; 131(2) : 187-91.

5- friedman M. : Thyroid carcinoma. Otolar Clin Am : 1986 ; 19(3) : 451-461.

6 -wada N, Quan-Yang D. lymph node metastasis from 259 papillary thyroid microcarcinomas, frequency, pattern of occurrence, and optimal strategy for neck dissection. Ann Surg : $2003 ; 237$ (3) : 399-407.

7- Abdelkafi M, Methloithi J, Ben Ali S et al. : Microcarcinomes thyroïdiens à propos de 14 cas J. Tun ORL . $2003 ; 10: 8-11$

8-Mathonnet M. : chirurgie ganglionnaire des cancers thyroïdiens différenciés non médullaires. Annales de chirurgie : $2006 ; 131: 361-8$.

9-Toumiaire J, Bernard M. H, Bizollon-Roblin et al. Le microcarcinome papillaire du corps de la thyroide. La presse médicale : $1998 ; 27(29)$ : 1467-9.

10-Harvey RD, Matheson MA, Grabowsky PA et al. : Measurement of serum thyreglobulin is of value in detecting tumor recurrence following treatment of differentiated thyroid carcinoma by lobectomy. Br J Surg. : 1990; 77(3) : 324-6.

11-David J. M, Ruaux Ch. : Multifocalité et lymphophilie des microcarcinomes papillaires thyroïdiens, résultats de la thyroidectomie totale avec évidements bilatéral à propos de 38 patients. Ann. Oto Laryng. (Paris) : 1992 ; 109 : 183-7. 12-Rodriguez JM, Moreno A. : papillary thyroid microcarcinoma clincal study and prognosis. Eur J Surg. : 1997 ; 163(4) : 255-9.

13-Kasai N, SakamotoA. : New subgrouping of ssmall thyroid carcinomas : cancer $1987 ; 15 ; 620(8): 1767-70$.

14- Ito $Y$, Uruno T, Nakano K, Takamura $Y$ et al : An observation trial without surgical treatment in patients with papillary microcarcinoma of the thyroid. Thyroid : $2003 ; 13(4): 381-7$

15-Mc Gregor GI : Lymph node metastases for well differentiated thyroid cancer. Am J Surg : $1985 ; 149: 610-612$.

16-Poletti EM, Narno Rossi G, D’Alioa G. : La terapia del carcinoma differnziato della tiroide. Chirurgia : $1994 ; 7: 315-18$

17-Morone G, Meriggi F, Forni E. : elementi di diagnostica a principi di terabit nel canro della tiroide : esperenza pavese 100anni dopo Bottini. G. Chir : 1992 ; 13:193-298.

18-Block M.A, Miller J. M, Horn R. C. : Thyroid carcinoam with cervical lymph node metastasis. Am J Surg. : $1971 ; 122(4): 458-63$.

19-George H. S, Giotakis J, Stafyla V. : Papillary thyroid microcarcinoma : a surgical perspective. Cancer Treat Rev. $2005 ; 31(6)$ : 423-38.

20- El araj. D.M ; Sturgeon C. : Treatment management papillary carcinomas of thyroïde gland The surgeon.2009;7(5): 286-9.

21- Sakorafos G.H ; Sampanis D ; Safileas M. : Cervcial lymph node dissection in papillary carcinomas of thyroîd gland.

Surgical Oncology. 2010;19(2): 57-70 